\title{
Surface analysis of magnetite nanoparticles in cyclohexane solutions of oleic acid and oleylamine
}

\author{
M. Klokkenburg *, J. Hilhorst, B.H. Erné \\ Van't Hoff Laboratory for Physical and Colloid Chemistry, Science Faculty, Utrecht University, Padualaan 8, 3584 CH Utrecht, The Netherlands \\ Received 28 April 2006; received in revised form 19 September 2006; accepted 19 September 2006 \\ Available online 20 October 2006
}

\begin{abstract}
The surface of magnetite $\left(\mathrm{Fe}_{3} \mathrm{O}_{4}, d=6.3 \pm 0.7 \mathrm{~nm}\right)$ nanoparticles dispersed in cyclohexane was studied in the presence of oleic acid and oleylamine using in situ FTIR spectroscopy. Equimolar mixtures of these surfactants are widely used in the chemical synthesis of nanoparticles with a low polydispersity. Here, the IR spectra indicate that oleic acid molecules adsorb to the magnetite surface as a carboxylate. Measurements as a function of surfactant concentration yield an adsorption isotherm, with about two surfactant molecules adsorbed per nm ${ }^{2}$ of magnetite at $1 \mathrm{mM}^{2}$ surfactant concentration and about 3.5 molecules per $\mathrm{nm}^{2}$ at $310 \mathrm{mM}$, of the order expected for full monolayer coverage. No spectral indication is found of oleylamine molecules at the surface of magnetite. In solution, however, almost every oleylamine molecule combines with an oleic acid molecule to form an acid-base complex, with an association constant of $3.5 \times 10^{4} \mathrm{dm}^{3} \mathrm{~mol}^{-1}$.
\end{abstract}

(C) 2006 Elsevier B.V. All rights reserved.

Keywords: Adsorption; Magnetite; Oleic acid; Oleylamine; In situ FTIR; Nanoparticles

\section{Introduction}

Inorganic nanoparticles whose surface is covered with surfactants like oleic acid can generally be dispersed in apolar solvents. The polar head of the surfactant molecules adsorbs to the nanoparticle surface, and the apolar tail of the surfactant is soluble. Many chemical routes for the synthesis of nanoparticles rely on varying the type and concentration of surfactants to tune the size of the nanoparticles obtained. A much-used surfactant for magnetic nanoparticles in non-aqueous ferrofluids is oleic acid [1], but recently the equimolar combination of oleic acid with oleylamine has been advocated [2-4]. Oleylamine affects the synthesis of nanoparticles of various materials, including FePt [2], FeMo [3], and magnetite $\left(\mathrm{Fe}_{3} \mathrm{O}_{4}\right)$ [3,5], although the mechanism remains unclear. Moreover, surfactant desorption is expected to take place during the chemical purification steps at the end of the synthesis, but few studies mention quantitative information about this problem [6,7]. Surfactant adsorption on nanoparticles is an important issue, since it not only affects the colloidal stability but also the

\footnotetext{
* Corresponding author. Tel.: +31 30 2532888; fax: +31 302533870 .

E-mail address: m.klokkenburg@ @hem.uu.nl (M. Klokkenburg).
}

optical [8] and magnetic properties [9-11]. The magnetic properties of magnetite are at the basis of several ferrofluidic [12] and biomedical applications [13], including contrast enhancement of magnetic resonance imaging (MRI). Here, we use in situ FTIR spectroscopy to analyze the surface of magnetite nanoparticles that are dispersed in solutions of oleic acid and oleylamine in cyclohexane.

\section{Experimental}

A sterically stabilized colloidal dispersion of magnetite nanoparticles in cyclohexane was prepared according to a procedure previously described [4,5]. In this procedure, magnetite nanoparticles are synthesized in $20 \mathrm{~mL}$ phenyl ether by reduction of iron(III) acetylacetonate using 1,2hexadecanediol. Prior to the reaction, a surfactant mixture of pure oleic acid (cis-9-octadecenoic acid) and pure oleylamine (cis-1-amino-9-octadecene) is added. These hydrocarbons are added to stabilize the nanoparticles that are formed as the reactant mixture is heated to reflux for $30 \mathrm{~min}$. After cooling to room temperature, the particles are precipitated by adding ethanol (20 mL, p.a.) and using a permanent magnet $(0.1 \mathrm{~T})$. Subsequently, the supernatant is removed and the particles are again dispersed in $20 \mathrm{~mL}$ phenyl ether to repeat the above 


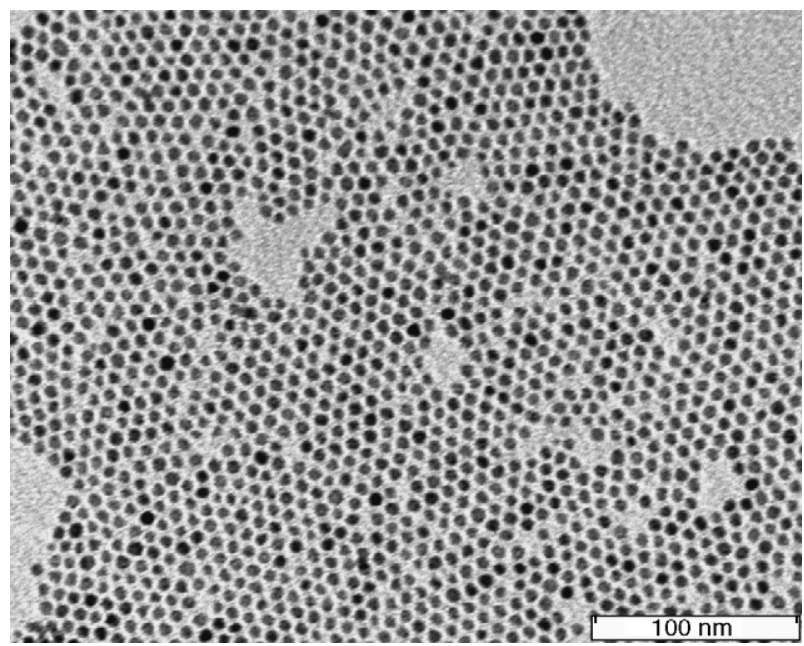

Fig. 1. Transmission electron microscopy (TEM) image of the investigated magnetite particles $(d=6.3 \pm 0.7 \mathrm{~nm})$.

described synthesis procedure twice. In this way, the average particle size is increased by the seeded growth of the particles prepared in the first step and the polydispersity is reduced from $33 \%$ to $11 \%$ [5]. After the last growth step, the supernatant is removed and the precipitated magnetite is dissolved in $5 \mathrm{~mL}$ cyclohexane. Finally, the dispersions are washed three times with $5 \mathrm{~mL}$ ethanol. In each purification step, the precipitates are dried using a $\mathrm{N}_{2}$ flow and redispersed in $5 \mathrm{~mL}$ pure cyclohexane.

Transmission electron microscopy images were obtained on a Philips Tecnai 10 TEM operating at $100 \mathrm{kV}$. The images show that the dispersion contains particles with an average size of 6.3 $\pm 0.7 \mathrm{~nm}$ (Fig. 1). Line broadening in X-ray diffraction patterns agrees with the particle diameter obtained from TEM, indicating that the particles are single crystals [5]. The magnetic diameter $(6.0 \mathrm{~nm})$ calculated from magnetization curves agrees with the physical diameter [14], indicating that the particles have a single magnetic domain [5]. In addition to the magnetic diameter, the particle concentration of the ferrofluid can be determined from magnetization curves. Based on the diameter, the surface area per gram magnetite is $186 \mathrm{~m}^{2}$.

Both in situ and ex situ FTIR spectroscopy were performed at $293 \mathrm{~K}$ to study the surfactant adsorption after purification. To this end, samples were prepared by first precipitating the particles $(0.4 \mathrm{~mL}$ ferrofluid) using $0.4 \mathrm{~mL}$ pure ethanol in the presence of a magnetic field of $0.1 \mathrm{~T}$. Subsequently, the precipitate was dried with $\mathrm{N}_{2}$ and the particles were redispersed in a 1:1 mixture of oleic acid and oleylamine $(0-320 \mathrm{mM})$ in $5 \mathrm{~mL}$ cyclohexane (Merck, spectroscopic grade) with a total magnetite concentration of $21 \mathrm{~g} / \mathrm{L}$.

Reference solutions without magnetic particles were prepared of 1:1 mixtures of oleic acid and oleylamine in cyclohexane ranging from 4 to $320 \mathrm{mM}$. Ex situ FTIR samples were prepared by precipitating $0.5 \mathrm{~mL}$ ferrofluid with $25 \mathrm{~mL}$ ethanol and centrifuging for $2 \mathrm{~h}$ at 18,000 rpm $(39,000 \times g)$ in an Avanti j-20 XP ultracentrifuge with a JA 25-50 rotor (Beckman Coulter). Both sediment and supernatant were first quantitatively transferred to $260 \mathrm{mg}$ potassium bromide (Fluka, spectroscopic grade), then heated in air for an hour at $80{ }^{\circ} \mathrm{C}$ to remove traces of solvent, and finally pressed into pellets. Prior to mixing, potassium bromide was vacuum dried at $80{ }^{\circ} \mathrm{C}$ for $10 \mathrm{~h}$ in order to reduce levels of absorbed water.

All in situ measurements were conducted using a $\mathrm{NaCl}$ fluid cell with a sample solution thickness of $100 \mu \mathrm{m}$ and with the incoming beam perpendicular to the cell. For both methods a Perkin-Elmer System 2000 FTIR infrared spectrometer was used for scanning in the range of $370-4000 \mathrm{~cm}^{-1}$ with a resolution of $1 \mathrm{~cm}^{-1}$ and an accumulation time of $5 \mathrm{~min}$.

\section{Results}

Surfactant solutions were first examined in the absence of magnetite nanoparticles. The IR spectra in Fig. 2 are of (a) cisoleic acid $(\mathrm{RCOOH}),\left(\right.$ b) cis-oleylamine $\left(\mathrm{RNH}_{2}\right)$, and (c) a $1: 1$ mixture of those surfactants dissolved in cyclohexane. They are differential spectra measured with cyclohexane as a reference, which explains the lack of signal at wavenumbers where cyclohexane strongly absorbs (indicated with grey bars). The spectra are presented in the ranges from 3300 to $2500 \mathrm{~cm}^{-1}$ and from 1800 to $600 \mathrm{~cm}^{-1}$, separated by two diagonal ticks on the

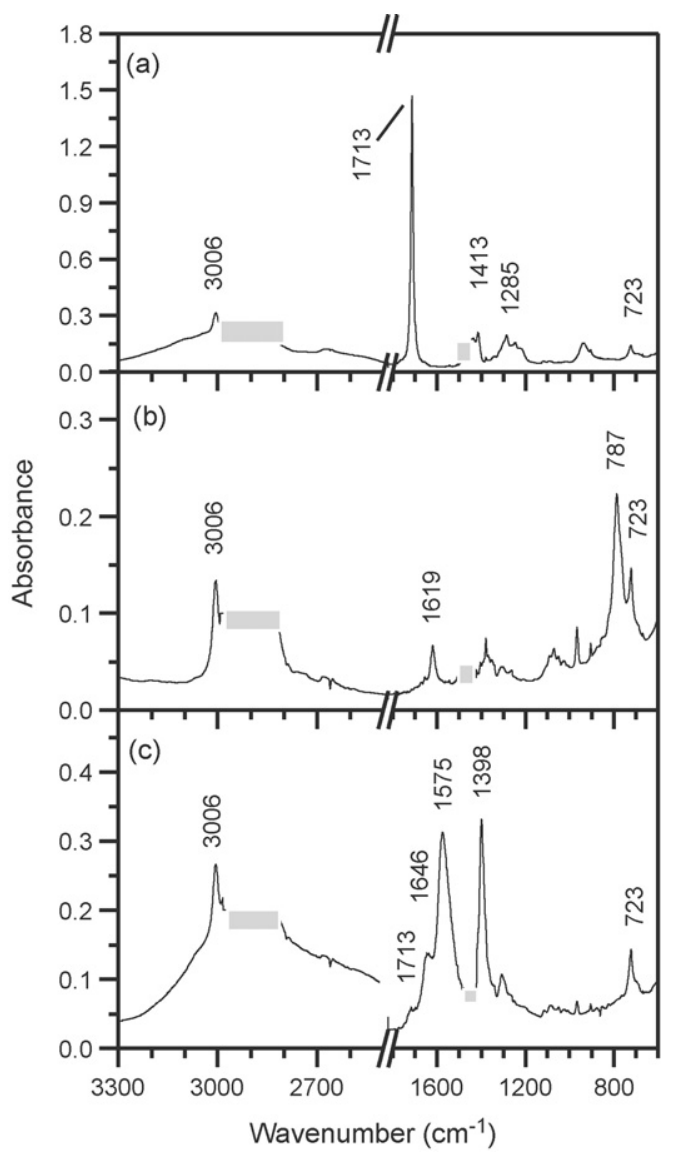

Fig. 2. Differential IR spectra of surfactant molecules dissolved in cyclohexane: (a) oleic acid (150 mM), (b) oleylamine (150 mM), and (c) a 1:1 mixture of oleic acid $(75 \mathrm{mM})$ and oleylamine $(75 \mathrm{mM})$. For clarity, parts of spectra (a)(c) where cyclohexane strongly adsorbs are removed with grey bars. The spectra are presented in the ranges from 3300 to $2500 \mathrm{~cm}^{-1}$ and from 1800 to $600 \mathrm{~cm}^{-1}$ with different vertical scales. Quantitative concentration dependences of peak absorbances are given in the text. 
Table 1

Assignment of the most relevant bands observed in the infrared spectra

\begin{tabular}{|c|c|c|}
\hline Wavenumber $\left(\mathrm{cm}^{-1}\right)$ & Assignment & Reference \\
\hline \multicolumn{3}{|l|}{ In situ (fluid cell) } \\
\hline 3006 & $\operatorname{cis} v(-\mathrm{CH}=)$ & {$[15-17]$} \\
\hline 1713 & $v(-\mathrm{C}=\mathrm{O})$ & {$[15-18]$} \\
\hline 1646 & $v(\mathrm{C}=\mathrm{C})$ & {$[15-18]$} \\
\hline 1619 & $\delta\left(\mathrm{NH}_{2}\right)$ & {$[16,19]$} \\
\hline 1575 & $v_{\mathrm{a}}\left(-\mathrm{COO}^{-}\right)$ & {$[15,18,20]$} \\
\hline 1413 & $\gamma(\mathrm{COH})$ & {$[21,22]$} \\
\hline 1398 & $v_{\mathrm{s}}\left(-\mathrm{COO}^{-}\right)$ & {$[15,23]$} \\
\hline 1285 & $v(\mathrm{C}-\mathrm{O})$ & [22] \\
\hline 1050 & Ethanol $v(\mathrm{C}-\mathrm{O})$ & \\
\hline 787 & $\gamma_{\mathrm{w}}\left(\mathrm{NH}_{2}\right)$ & \\
\hline 723 & $v(\mathrm{CH}=\mathrm{CH})$ & [17] \\
\hline 626 & $v(\mathrm{Fe}-\mathrm{O})$ & {$[7,24,25]$} \\
\hline \multicolumn{3}{|l|}{ Ex situ (KBr) } \\
\hline 3006 & $\operatorname{cis} v(-\mathrm{CH}=)$ & {$[15,26]$} \\
\hline 2954 & $v_{\mathrm{a}}\left(\mathrm{CH}_{3}\right)$ & {$[7,26]$} \\
\hline 2923 & $v_{\mathrm{a}}\left(\mathrm{CH}_{2}\right)$ & {$[15,27]$} \\
\hline 2852 & $v_{\mathrm{s}}\left(\mathrm{CH}_{2}\right)$ & {$[15,27]$} \\
\hline 1524 & $v_{\mathrm{a}}\left(-\mathrm{COO}^{-}\right)$ & {$[20,23]$} \\
\hline 1464 & $\delta\left(\mathrm{CH}_{2}\right)$ & {$[23,27]$} \\
\hline 1434 & $v_{\mathrm{s}}\left(-\mathrm{COO}^{-}\right)$ & {$[20,23]$} \\
\hline 1411 & $v_{\mathrm{s}}(\mathrm{C}-\mathrm{O})$ & {$[23,27]$} \\
\hline 1050 & Ethanol $v(\mathrm{C}-\mathrm{O})$ & \\
\hline 635 & $v_{1}(\mathrm{Fe}-\mathrm{O})$ & {$[24,27,28]$} \\
\hline 590 & $v_{2}(\mathrm{Fe}-\mathrm{O})$ & {$[24,27,28]$} \\
\hline
\end{tabular}

horizontal scale bar, to show more clearly the most relevant bands (there are no new specific bands from 2500 to $1800 \mathrm{~cm}^{-1}$ ). Note that the most relevant peak assignments of both in situ and ex situ spectra are presented in Table 1. The spectra of the pure surfactants are well known from literature $[15,18,22]$. A common feature is the olefinic $\mathrm{C}-\mathrm{H}$ stretching mode at $3006 \mathrm{~cm}^{-1}$ from the backbone of the surfactant molecules (Fig. $2 \mathrm{a}$ and b). Specific to the oleic acid spectrum is the carbonyl absorbance at $1713 \mathrm{~cm}^{-1}$, a position that indicates that the molecules are present in dimers that are held together by hydrogen bonding $[15,18]$. In the oleylamine spectrum the most characteristic peak is the $787 \mathrm{~cm}^{-1}$ absorbance which can be ascribed to a $\mathrm{NH}_{2}$ wagging vibration. The main new spectral features of the 1:1 mixtures are the signals at 1575 and $1398 \mathrm{~cm}^{-1}$, respectively, the asymmetric and the symmetric stretching modes of a carboxylate. They arise from the acid-base complex of an oleic acid molecule with an oleylamine molecule:

$$
\mathrm{RCOOH}+\mathrm{RNH}_{2} \rightleftharpoons \mathrm{RCOO}^{-}: \mathrm{RNH}_{3}{ }^{+}
$$

The broad band from 3200 to $2000 \mathrm{~cm}^{-1}$ is ascribed to $\mathrm{H}$ bonding in the complex.

A quantitative analysis was performed of spectra as presented in Fig. 2 as a function of concentration. From this, the equilibrium constant for the formation of the acid-base complex (Eq. (1)) could be obtained in the following way. The absorbance $A_{3006}$ at $3006 \mathrm{~cm}^{-1}$ was taken as a measure of the total concentration of surfactants in the system: $A_{3006}=C_{3006} \times\left[\mathrm{RCOOH}+\mathrm{RNH}_{2}\right]$, where $C_{3006}=(4.57 \pm 0.025) \times 10^{-4} \mathrm{dm}^{3} \mathrm{mmol}^{-1}$ and where $\left[\mathrm{RCOOH}+\mathrm{RNH}_{2}\right]$ is the total surfactant concentration in mmol dm ${ }^{-3}$. The concentration of "free" oleic acid (molecules not involved in an acid-base complex) was determined from the absorbance at $1713 \mathrm{~cm}^{-1}: A_{1713}=C_{1713} \times[\mathrm{RCOOH}]$, where $C_{1713}=8.3 \times 10^{-3} \mathrm{dm}^{3} \mathrm{mmol}^{-1}$ and where $[\mathrm{RCOOH}]$ is the free oleic acid (in dimers) concentration in $\mathrm{mmol} \mathrm{dm}^{-3}$. The concentration of free oleylamine was taken to be equal to the free oleic acid concentration, because equimolar systems had been prepared across the 4-320 $\mathrm{mM}$ range. The expected quadratic dependence of the concentration of the complex on the free oleic acid concentration was confirmed. The equilibrium constant $K_{\text {[acid-base] }}$ that describes the acid-base equilibrium was found to be $K_{\text {[acid-base] }}=c_{\mathrm{ab}} /\left(c_{\mathrm{a}} \times c_{\mathrm{b}}\right)=(346 \pm 4) \times 10^{2} \mathrm{dm}^{3} \mathrm{~mol}^{-1}$, where $c_{\mathrm{ab}}$ is the concentration of $\mathrm{RCOOH}+\mathrm{RNH}_{2}$ complex, $c_{\mathrm{a}}$ the concentration of free oleic acid and $c_{\mathrm{b}}$ the concentration of free oleylamine. The equilibrium strongly favors complex formation.

In the presence of magnetite nanoparticles in solution (see Fig. 3), a strong new band appears at $626 \mathrm{~cm}^{-1}$ that can be attributed to vibrations of the crystalline lattice of nanocrystalline magnetite [7,24,25]. At low surfactant concentration (Fig. 3a), the spectra indicate the presence of adsorbed carboxylate groups with a symmetric mode around $1420 \mathrm{~cm}^{-1}$ and an asymmetric mode at $1545 \mathrm{~cm}^{-1}[15,23]$. At high surfactant concentration (Fig. 3b), this absorption is overshadowed by that of the acid-base complex in solution (see

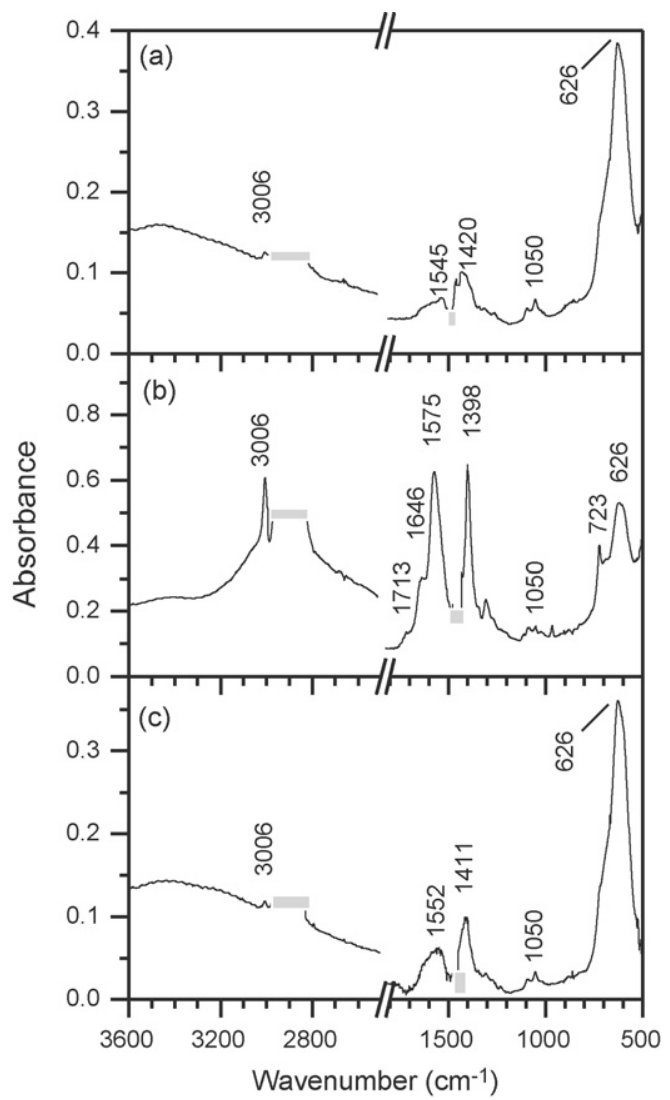

Fig. 3. Differential IR spectra of magnetite nanoparticles dispersed in cyclohexane in the presence of 1:1 mixtures of oleic acid and oleylamine: (a) at $1 \mathrm{mM}$ surfactant concentration, (b) at $320 \mathrm{mM}$ surfactant concentration, and (c) also at $320 \mathrm{mM}$ surfactant concentration, but after subtraction of the contribution of non-adsorbed surfactant species. 
Fig. 2c). The amount of that complex can be determined on the basis of the $3200-2000 \mathrm{~cm}^{-1}$ band, so that the contribution of solution species can be subtracted assuming no free oleic acid or oleylamine in solution. This results in Fig. 3c, which resembles the spectrum with low excess surfactant concentration (Fig. 3a). The shift from $1420 \mathrm{~cm}^{-1}$ in Fig. 3a to $1411 \mathrm{~cm}^{-1}$ in Fig. $3 \mathrm{~b}$ is probably still due to a small contribution of non-adsorbed surfactant species (Fig. 3b), as a result of a small error in the determination of the amount of non-adsorbed surfactant species using the $3200-2000 \mathrm{~cm}^{-1}$ band. The weak absorbance at $1713 \mathrm{~cm}^{-1}$ in Fig. $3 \mathrm{~b}$ can fully be ascribed to free oleic acid, because its strength agrees with the concentration of acid-base complex and the earlier determined association constant. No spectral feature is found that is characteristic of an amine-containing species in the spectra of adsorbed surfactant (Fig. 3a and b). The peaks around $1050 \mathrm{~cm}^{-1}$ are due to a remnant of ethanol from the chemical purification step.

An ex situ spectrum of sedimented particles is shown in Fig. 4a, which is quite similar to the in situ spectra of the particles (Fig. 3a). The spectrum of the sedimented particles clearly indicates the presence of magnetite nanoparticles by the broad band around $590 \mathrm{~cm}^{-1}$ (Table 1) and the methylene stretch vibration at $3006 \mathrm{~cm}^{-1}$ of the adsorbed surfactants. In addition, a complex series of absorbance bands is observed between 1400 and $1600 \mathrm{~cm}^{-1}$, among which the stretching modes of the carboxylate groups of the surfactant molecules. However, comparing this spectrum with reference spectra of the free surfactants and the complex in $\mathrm{KBr}$, there is no indication of free surfactants that are not adsorbed to the surface of magnetite. The broad band around $3400 \mathrm{~cm}^{-1}$ and the peak at $1650 \mathrm{~cm}^{-1}$ point to trace amounts of water in the potassium bromide.

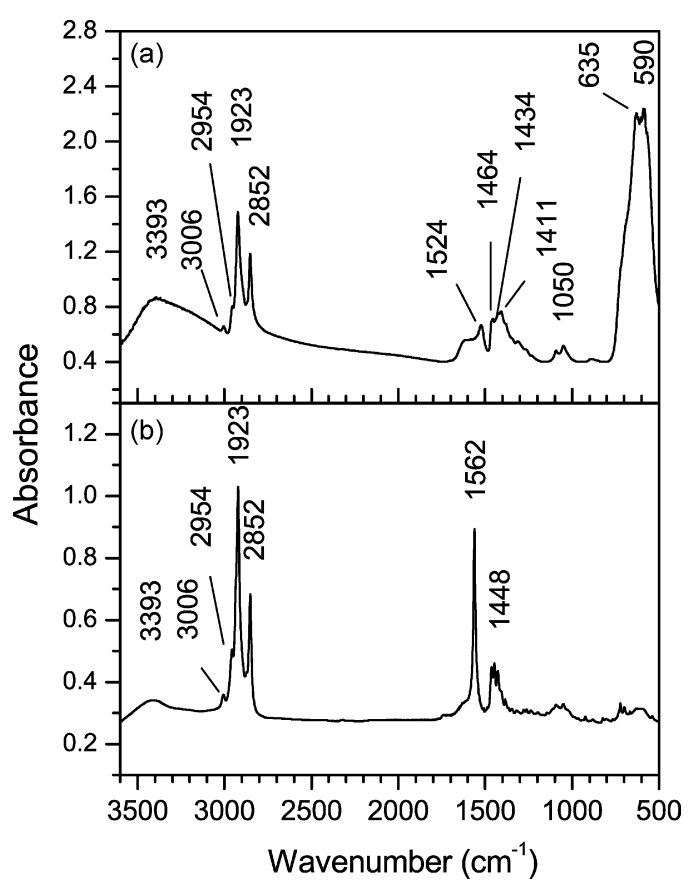

Fig. 4. Ex situ KBr spectra obtained after ultracentrifugation: (a) the sedimented particles and (b) the species present in the supernatant after evaporation of the solvent.
The surface coverage of the magnetite nanoparticles was determined as a function of the surfactant concentration in solution, for 1:1 mixtures of oleic acid and oleylamine. A measure of the amount of complex in solution was given by the $3200-2000 \mathrm{~cm}^{-1}$ band. On this basis, the $1398 \mathrm{~cm}^{-1}$ absorbance by dissolved acid-base complex was calculated and subtracted from the measured absorbance. The remaining absorbance was taken as a relative measure for the amount of adsorbed surfactant. It was converted to an absolute amount of surfactant on the basis of in situ spectra of particles dispersed in pure cyclohexane. On the basis of in situ spectra the ratio could be determined between $1420 \mathrm{~cm}^{-1}$ absorbance (adsorbed surfactants) and $3006 \mathrm{~cm}^{-1}$ absorbance, for which the absolute calibration was given earlier. The total magnetite surface area was calculated from the volume of magnetite and the diameter of the particles. The volume of magnetite was calculated from the absorbance by magnetite nanocrystals at $626 \mathrm{~cm}^{-1}$, which was found to be 0.36 at the concentration of $21 \mathrm{~g} / \mathrm{L}$.

The concentration dependence of the adsorbed amount of surfactants (Fig. 5) can be described by a Langmuir isotherm [29], which describes the adsorption to the weak adsorption sites, and by a constant corresponding to adsorption to strong adsorption sites. The Langmuir isotherm is defined as $\theta=K x_{\text {sol }} /\left(1+K x_{\text {sol }}\right)$, where $\theta$ is the filled fraction of adsorption sites that are free at $1 \mathrm{mM}$ surfactant concentration $\left(\theta=1\right.$ at $\left.5.9 \mu \mathrm{mol} \mathrm{m} \mathrm{m}^{-2}\right), x_{\text {sol }}$ the total mole fraction of surfactants in solution, and $K$ is the equilibrium constant. The strong adsorption sites are all occupied above $1 \mathrm{mM}$ and correspond to $3.4 \mu \mathrm{mol} \mathrm{m}{ }^{-2}$ (two surfactant molecules per $\left.\mathrm{nm}^{2}\right)$. An estimate of $-5 k_{\mathrm{B}} T(-12 \mathrm{~kJ} / \mathrm{mol})$ can be given for the adsorption free energy $F_{\text {ads }}$, using $K=\mathrm{e}^{-F_{\text {ads }} / k_{\mathrm{B}} T}=115$ from a fit of the adsorption isotherm. This adsorption free energy is of the same order as the adsorption energies calculated by Korolev et al. [7] from temperature-dependent adsorption data for oleic acid on magnetite in hexane. For the strong adsorption sites, the adsorption free energy is significantly larger than $-5 k_{\mathrm{B}} T$.

The error bars in Fig. 5 are not determined by noise but they estimate a systematic error in the determination of the baseline of the $3200-2000 \mathrm{~cm}^{-1}$ band. A second series of infrared

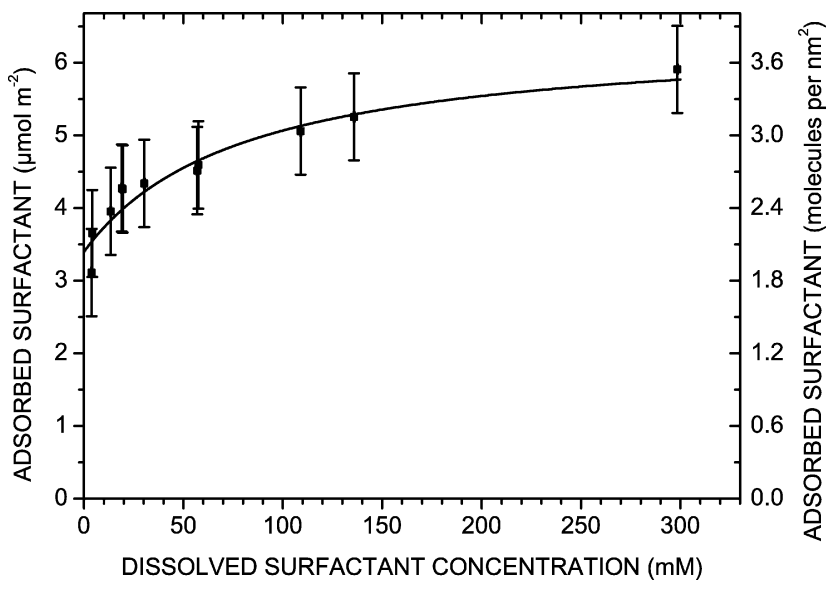

Fig. 5. Adsorption on the surface of magnetite nanoparticles in cyclohexane as a function of the surfactant concentration of 1:1 mixtures of oleic acid and oleylamine. 
measurements was performed with the same magnetite particles but at lower concentrations, affecting the contribution of light scattering by the magnetite particles to the baseline absorbance and revealing that a systematic error in its determination was the limiting factor for the accuracy of the adsorption isotherm.

Surfactant desorption during chemical washing steps was studied as follows. Ethanol was added to destabilize the dispersion, followed by redispersal in cyclohexane. The dispersions were still stable at 50 vol.\% ethanol, even in a centrifuge at an acceleration of $1425 \times g$, and they became abruptly unstable around 60 vol.\% ethanol. For comparison, hexadecane is miscible with cyclohexane and has a solubility of about $1 \mathrm{M}$ at $60 \mathrm{vol} \%$ ethanol and about $0.7 \mathrm{M}$ in pure ethanol (hexadecane resembles the non-polar chain of the surfactant molecules). After addition of $50 \mathrm{~mL}$ of ethanol per $\mathrm{mL}$ of washed (three times) dispersion and after centrifugation for $2 \mathrm{~h}$ at an acceleration of $39,000 \times g$, about one third of the surfactant desorbed, yielding a $0.1 \mathrm{mM}$ surfactant concentration in solution. This indicates that, in contrast to what is suggested in Fig. 5, significant surfactant desorption may still occur when the concentration is decreased below $1 \mathrm{mM}$; desorption of one third of the molecules upon going from 1 to $0.1 \mathrm{mM}$ suggests that the strong adsorption sites have an adsorption energy of at least $-25 \mathrm{~kJ} / \mathrm{mol}$ (using $\Delta \theta \approx K \Delta x_{\mathrm{sol}}$ ). In the spectrum of the supernatant (Fig. 4b), obtained by quantitative transfer to $\mathrm{KBr}$ and evaporation of the solvent, two peaks at 1562 and $1448 \mathrm{~cm}^{-1}$ can be observed, indicating predominantly oleate species [20,23]. Since no evidence is found for the presence of oleylamine in the supernatant, this suggests that oleylamine has been removed in previous purification steps and that oleic acid is the dominant species in the adsorbed layer. It is remarked that surface desorption also seems to occur upon high dilution in pure cyclohexane, as is revealed by the lower stability of dilute systems over periods of a few months.

\section{Discussion}

The surface of magnetite nanoparticles dispersed in cyclohexane was analyzed by IR spectroscopy in the presence of oleic acid and oleylamine surfactants. We now discuss the amount of surfactant that adsorbs, the role of oleylamine, and the practical implications for the chemical preparation of magnetite nanoparticles.

The amount of 2-3.5 adsorbed surfactant molecules per $\mathrm{nm}^{2}$ can be compared to literature and to calculations on the basis of the size of the nanoparticles and the surfactant molecules. Dubois et al. [6] used chemical micro-analysis to study the adsorption of oleic acid without the presence of oleylamine on maghemite particles, which were initially prepared in aqueous solutions and subsequently transferred to cyclohexane. They found a plateau value of about $3.5 \pm 0.4$ adsorbed molecules per $\mathrm{nm}^{2}$ at $10 \mathrm{mM}$ oleic acid. Korolev et al. [7] used in situ IR analysis to study the adsorption of oleic acid on magnetite particles (prepared in water) in hexane solutions; they do not give absolute coverages, but the surface coverage attains a plateau in about the same concentration range as in our measurements. Surfactant adsorption apparently strongly depends on how the nanoparticles are prepared, since a plateau is reached at significantly lower concentration by Dubois et al. [6], and complete desorption is observed at low surfactant concentrations by Korolev et al. [7], in contrast to our findings and those of Dubois et al. [6]. The presence of a fractional monolayer is supported by other techniques: both from statistical analysis of the particle-particle contact distance observed by TEM [11] and from small angle neutron scattering (SANS) [30], a surfactant layer of about $2 \mathrm{~nm}$ thickness was deduced. This agrees with the layer thickness expected from the size of the surfactant molecules on the basis of monolayer coverage of the as-prepared nanoparticles by surfactant molecules attached perpendicular to the surface. At full surface coverage, a 2-nm-thick shell of oleic acid would correspond to about 3.8 molecules per $\mathrm{nm}^{2}$, assuming that the adsorbed surfactant volume is equal to the surface area of the magnetite multiplied by $2 \mathrm{~nm}$ (assuming that every surfactant molecule is anchored to the surface). This estimate is close to our experimental values and those of Dubois et al. [6]. From this, it can be concluded that a large part of the surface is covered with adsorbed surfactant molecules.

The effect of oleylamine on the adsorption of oleic acid on magnetite is not obvious. On the one hand, the spectra do not give any clear indication of the presence of oleylamine molecules at the surface of magnetite (Figs. 3 and 4). On the other hand, the same can be said of the spectra of the acid-base complex, where despite the lack of direct spectral evidence, no doubt exists about the presence of oleylamine (Fig. 2). Ex situ IR analysis of FePt nanoparticles prepared with oleic acid and oleylamine suggest the presence of oleylamine molecules at the surface, from absorption at 3300 and $1593 \mathrm{~cm}^{-1}$ [16]. However, this does not mean that the same peak positions are necessarily expected here, nor that surfactant adsorption has to be the same on magnetite. Adsorption of oleic acid on silica, for instance, is completely different, with hydrogen bonding to surface $\mathrm{Si}-\mathrm{OH}$-groups rather than the formation of a carboxylate [31-33].

A chemical argument against the presence of oleylamine molecules in the surface layer is that it is unclear how it could bind to magnetite. Moreover, an oleic acid molecule cannot at the same time form a carboxylate with oleylamine and with the magnetite surface. In the preparation of FeMo nanoparticles, a 1:1 mixture of oleic acid and oleylamine yields significantly smaller particles than either surfactant on its own, suggesting stronger surfactant adsorption [34]. The explanation that was proposed [34] is that due to acid-base complex formation with oleylamine, many more oleic acid molecules have a dissociated $\mathrm{OH}$-group than with oleic acid alone, and that this promotes adsorption. Another argument could be that when $\mathrm{RCOOH}$ adsorbs and becomes $\mathrm{RCOO}^{-}$, an $\mathrm{H}^{+}$is released that must somehow be neutralized, for instance by oleylamine, creating $\mathrm{RNH}_{3}{ }^{+}$, although neutralization could also be obtained by the release of an adsorbed $\mathrm{OH}$-group. Our adsorption isotherm does not show stronger adsorption of oleic acid than in studies without oleylamine $[6,7]$. It may be that the only reason why use of oleylamine in combination with oleic acid is advocated with magnetite is that this has been found to work well with 
other nanomaterials, and that the influence of including oleylamine in the synthesis of magnetite has not been studied systematically.

A practical implication of our findings is that oleic acid is still able to desorb from magnetite even when 1:1 mixtures with oleylamine are used. Desorption therefore also occurs during chemical purification steps of the synthesis. In such steps, nanoparticles are separated from the solvent and excess reaction products by addition of polar solvent like ethanol or acetone, which causes precipitation of the nanoparticles. The polar head groups of the adsorbed surfactants are in contact with the nanoparticle surface, whereas the apolar chains are in contact with the solution. Adsorbed surfactants do not dissolve as well after the addition of polar solvent, but desorbed surfactant molecules still do. It was verified that both oleic acid and oleylamine can be dissolved in pure ethanol at molar concentrations. This means that when the nanoparticles are washed using too much pure ethanol, significant desorption of surfactants may occur. If too much desorbs, irreversible aggregation might occur due to Van der Waals attraction between the nanoparticles. From the adsorption isotherm, it appears that the addition of 1 to $10 \mathrm{mM}$ surfactants in all washing steps is recommended, to limit desorption without polluting the system with excessive amounts of non-adsorbed surfactant.

\section{Conclusion}

It is unclear whether oleylamine is present in the surfactant layer adsorbed on magnetic nanoparticles dispersed in cyclohexane in the presence of oleic acid and oleylamine. What is clear, however, is that oleylamine forms an acid-base complex with oleic acid in the solution and that oleylamine does not prevent desorption of surfactant upon dilution. This study illustrates one of the strengths of in situ IR spectroscopy in the study of surfactant adsorption on nanoparticles. Different chemical species, in solution, at the surface of nanoparticles, and in the crystalline core of the nanoparticles, can be analyzed simultaneously and quantitatively. An advantage of nanoparticles is the high surface area per gram of material, so that the absorbance of surface species is relatively strong.

\section{Acknowledgements}

Fouad Soulimani and Tom Visser are thanked for their help with the infrared measurements. Willem Kegel is thanked for fruitful discussions.

\section{References}

[1] R. Massart, IEEE Trans. Magn. MAG-17 (1981) 1247.

[2] S.H. Sun, C.B. Murray, D. Weller, L. Folks, A. Moser, Science 287 (2000) 1989.

[3] S.H. Sun, H. Zeng, D.B. Robinson, S. Raoux, P.M. Rice, S.X. Wang, G.X. Li, J. Am. Chem. Soc. 126 (2004) 273.

[4] S.H. Sun, H. Zeng, J. Am. Chem. Soc. 124 (2002) 8204.

[5] M. Klokkenburg, C. Vonk, E.M. Claesson, J.D. Meeldijk, B.H. Erné, A.P. Philipse, J. Am. Chem. Soc. (2004) 16706.

[6] E. Dubois, V. Cabuil, F. Boue, R. Perzynski, J. Chem. Phys. 111 (1999) 7147.

[7] V.V. Korolev, A.G. Ramazanova, A.V. Blinov, Russ. Chem. Bull. 51 (2002) 2044

[8] N.J. Turro, P.H. Lakshminarasimhan, S. Jockusch, S.P. O’Brien, S.G Grancharov, F.X. Redl, Nano Lett. 2 (2002) 325.

[9] S.W. Charles, in: J. Dormann, D. Fionari (Eds.), Studies of Magnetic Properties of Fine Particles and their Relevance to Materials Science, Elsevier, Amsterdam, 1992.

[10] R.H. Kodama, J. Magn. Magn. Mater. 200 (1999) 359.

[11] M. Klokkenburg, R.P.A. Dullens, W.K. Kegel, B.H. Erné, A.P. Philipse, Phys. Rev. Lett. 96 (2006) 037203.

[12] K. Raj, R. Moskowitz, J. Magn. Magn. Mater. 85 (1990) 233.

[13] Q.A. Pankhurst, J. Connolly, S.K. Jones, J. Dobson, J. Phys. D: Appl. Phys. 36 (2003) 167.

[14] B.H. Erné, K. Butter, B.W.M. Kuipers, G.J. Vroege, Langmuir 19 (2003) 8218.

[15] D.H. Lee, R.A. Condrate, J. Mater. Sci. 34 (1999) 139.

[16] N. Shukla, C. Liu, P.M. Jones, D. Weller, J. Magn. Magn. Mater. 266 (2003) 178.

[17] G.G. Sinclair, A.F. McKay, G.S. Myers, R.N. Jones, J. Am. Chem. Soc. 74 (1952) 2578

[18] D.H. Lee, R.A. Condrate, W.C. Lacourse, J. Mater. Sci. 35 (2000) 4961.

[19] W. Erley, J.C. Hemminger, Surf. Sci. 316 (1994) L1025.

[20] J.A. Mielczarski, J.M. Cases, P. Tekely, D. Canet, Langmuir 9 (1993) 3357.

[21] P. Thistlethwaite, M. Gee, D. Wilson, Langmuir 12 (1996) 6487.

[22] Y. Hou, H. Kondoh, M. Shimojo, E. Sako, N. Ozaki, T. Kogure, T. Ohta, J. Phys. Chem. B 109 (2005) 4845.

[23] M. Gasgnier, J. Mater. Sci. Lett. 20 (2001) 1259.

[24] L. Yu, L. Zheng, J. Yang, Mater. Chem. Phys. 66 (2000) 6.

[25] B. Grzeta, M. Ristic, I. Nowik, S. Music, J. Alloy. Compd. 334 (2002) 304.

[26] P. Thistlethwaite, M. Gee, D. Wilson, Langmuir 12 (1996) 6487.

[27] X.Q. Xu, H. Shen, J.R. Xu, X.J. Li, Appl. Surf. Sci. 221 (2004) 430.

[28] M. Yamaura, R.L. Camilo, L.C. Sampaio, M.A. Macedo, M. Nakamura, H.E. Toma, J. Magn. Magn. Mater. 279 (2004) 210.

[29] R.J. Hunter, Foundations of Colloid Science, 2nd ed., Oxford University Press, New York, 2001, pp. 277-303.

[30] M. Klokkenburg, J.D. Meeldijk, B.H. Ern,, A. Wiedenmann, A. Petukhov, R.P.A. Dullens, A.P. Philipse, Phys. Rev. Lett. (2006), in press.

[31] K. Marschall, H. Rochester, J. Chem. Soc., Faraday Trans. I 71 (1975) 1754.

[32] G. Blyhoder, C. Adhikar, A. Proctor, Colloid Surf. 105 (1995) 151.

[33] Z.W. Li, Y.F. Zhu, Appl. Surf. Sci. 211 (2003) 315.

[34] Y. Li, J. Liu, Y.Q. Wang, Z.L. Wang, Chem. Mater. 13 (2001) 1008. 